\title{
PERFIL EPIDEMIOLÓGICO, CLÍNICO E TERAPÊUTICO DE PACIENTES EM PÓS- OPERATÓRIO DE ESTOMAS DE ALIMENTAÇÃO
}

\section{EPIDEMIOLOGICAL, CLINICAL AND THERAPEUTIC PROFILE OF PATIENTS IN POSTOPERATIVE FEEDING STOMA}

\author{
Rizocele da Silva Souza ${ }^{1} *$ Alana Tamar Oliveira de Sousa ${ }^{2} *$ Karla Karolline Barreto $^{*}$ \\ Cardins $^{3 *}$ Edlene Régis Silva Pimentel $^{4}$
}

\begin{abstract}
RESUMO
Objetivos: Descrever o perfil sociodemográfico, clínico e terapêutico de pacientes em pós-operatório de estomas de alimentação de um hospital escola. Específicos: Caracterizar os pacientes conforme dados sociodemográficos; identificar as principais indicações, técnica operatória utilizada, tipos de sondas empregadas e dietas em pacientes em pós-operatório de estomas de alimentação; reconhecer as complicações pós-operatórias e o desfecho de pacientes submetidos a estomas de alimentação. Métodos: Estudo documental-descritivo, com abordagem quantitativa, realizada num hospital escola. Os dados foram analisados a partir do pacote estatístico SPSS (Statistical Package for Social Sciences) versão 21.0. Foram obtidas distribuições absolutas e percentis e as medidas estatísticas médias e desvio padrão de técnicas de estatística descritiva. Resultados: O público da amostra era composto na maioria por homens, aposentados e agricultores, residentes na zona urbana, com renda per capita baixa, ex-etilistas e ex-tabagistas, tendo indicação da gastro/jejunostomia para suporte nutricional. As complicações pós-operatórias foram tanto locais quanto sistêmicas; com tempo internação prolongados, porém com desfecho para alta hospitalar. Conclusão: Os pacientes apresentaram inúmeras complicações pós-operatórias, de origem multifatorial.

Palavras chave: Estomas Cirúrgicos. Gastrostomia. Jejunostomia. Cuidados de Enfermagem. Complicações Pós-Operatórias. Estomaterapia.
\end{abstract}

\begin{abstract}
Objectives: To describe the sociodemographic, clinical and therapeutic profile of patients in the postoperative period of stomata feeding at a teaching hospital. Specific: Characterize patients according to sociodemographic data; identify the main indications, operative technique used, types of tubes used and diets in patients in the postoperative period of stoma feeding; to recognize postoperative complications and the outcome of patients undergoing stomata feeding. Methods: Documentary-descriptive study, with a quantitative approach, carried out in a teaching hospital. The data were analyzed using the statistical package SPSS (Statistical Package for Social Sciences) version 21.0. Absolute distributions and percentiles were obtained, as well as the mean statistical measures and standard deviation of descriptive statistics techniques. Results: The sample population was composed mostly of men, retirees and farmers, living in the urban area, with low per capita income, ex-alcoholics and ex-smokers, with indication of gastro / jejunostomy for nutritional support. Postoperative complications were both local and systemic; with prolonged hospital stay, but with outcome for hospital discharge. Conclusion: The patients presented numerous postoperative complications, of multifactorial origin.
\end{abstract}

Keywords: Surgical Stomas. Gastrostomy. Jejunostomy. Nursing Care. Postoperative Complications. Stomatherapy.

\footnotetext{
${ }^{1}$ Bacharel em enfermagem pela Universidade Federal de Campina Grande. Pós-graduada em enfermagem dermatológica pela Sociedade Brasileira de Enfermagem em Feridas e Estética em parceria com a Estácio de Sá. Orcid: 0000.0002-6242-0815. Email: rizocele.nf@hotmail.com

2 Doutora em enfermagem pela Universidade Federal da Paraíba-UFPB. Mestre em Enfermagem pela UFPB. Mestre em Enfermagem em terapia intensiva pela Sociedade brasileira de de terapia intensiva-SOBRATI. Especialista em enfermagem do trabalho pela faculdade integrada de Patos-FIP. Graduada em enfermagem pela UFPB. Docente do curso de graduação em enfermagem da Universidade Federal de Campina Grande-UFCG, campus Cuité. Membro do grupo de Estudos e pesquisa em tratamento de feridas- GEPEFE/UFPB e do Grupo de Estudos e Pesquisas Interdisciplinares em Saúde e EnfermagemGEPISE/UFCG. Especialização em andamento em enfermagem dermatológica pela Sociedade Brasileira de Enfermagem em Feridas e Estética em parceria com a Estácio de Sá. Orcid: 0000.0002-1683-2851

${ }^{3}$ Mestre em Saúde Pública pela Universidade Estadual da Paraíba-UFPB. Graduada em enfermagem pela Universidade Federal de Campina Grande-UFCG. Docente no curso de enfermagem na UFCG. 0000.0002-5571-2932

${ }^{4}$ Enfermeira graduada em bacharel em enfermagem pela Faculdade Santa Emília de Rodat). Especialista em Terapia Intensiva pela Faculdade de Ciências Médicas-FCM. Docente do curso de bacharelado em enfermagem da Universidade Federal de Campina Grande-UFCG, campus Cuité. Pós-graduanda em enfermagem dermatológica pela Universidade Estácio de Sá. 0000.0001-84410944
}

https://doi.org/10.31011/reaid-2021-v.95-n.34-art.999 Rev Enferm Atual In Derme v. 95, n. 34, 2021 e-021047 


\section{INTRODUÇÃO}

A terapia nutricional através de gastrostomia ou jejunostomia é comum na prática clínica. Ambas são cirurgias realizadas para acesso gástrico e entérico, respectivamente, nas quais são criadas um estoma para introdução de uma sonda para proporcionar alimentação ao paciente ${ }^{(1)}$, são introduzidas em substituição às sondas nasogástrica ou nasoentérica, para aqueles pacientes que necessitam de nutrição prolongada, sendo indicada a realização de uma gastrostomia ou jejunostomia. As principais causas para estes procedimentos são as doenças malignas do sistema gastrointestinal superior, tais como carcinoma epidermóide de esôfago, de faringe, de cavidade oral e de laringe, adenocarcinoma de transição esofagogástrica estenosante ${ }^{(2)}$. Estas, no entanto contribuem em parte, para o desfecho dos processos cirúrgicos com finalidade para alimentação.

O profissional de enfermagem como membro de uma equipe que assiste à saúde dos indivíduos pode reduzir o número destas complicações conforme cuidados adequados, tanto na inserção, remoção, ou manuseio da sonda de alimentação nas gastrostomias. Cuidados como: realizar educação continuada dos profissionais para manusear adequadamente a sonda, orientação para o paciente e/ou familiares; buscar a equipe multidisciplinar para avaliar o paciente antes mesmo da cirurgia; usar técnicas assépticas ao cuidar da pele periestoma a fim de evitar infecção; manter cuidados em relação ao preenchimento do balonete da sonda; realizar aspiração de conteúdo gástrico antes de administrar dieta (em gastrostomia de baixo perfil); podem minimizar danos aos pacientes, no entanto, existem poucos estudos na literatura sobre os cuidados destes indivíduos com a sonda de gastrostomia que muitas vezes evoluem para complicações.

Diante das inúmeras complicações que podem surgir nos pacientes quanto ao uso de gastrostomia ou jejunostomia, mostra-se a necessidade e a importância de pesquisa nesta área para diferentes contextos, tendo em vista ser um tema pouco trabalhado nos estudos de âmbito nacional, e mais comumente mostrado em artigos internacionais. É importante para área da saúde, pois pesquisas deste tipo são geradoras de novos conhecimentos, que podem auxiliar na prevenção de complicações, no tratamento e reabilitação de pacientes estomizados, podendo ainda proporcionar a adoção de boas práticas mediante o cuidado adequado com tais pacientes. Tem fundamental apreço para enfermagem, uma vez que é a categoria que assiste o paciente com seus cuidados, em todas as situações de comorbidades, inclusive, é a enfermagem a responsável por executar os cuidados com as feridas e estomas, a qual deve estar preparada para lidar com complicações dos estomas de alimentação, e até mesmo ofertar um cuidado pré e póshttps://doi.org/10.31011/reaid-2021-v.95-n.34-art.999 Rev Enferm Atual In Derme v. 95, n. 34, 2021 e-021047 
operatório que previna danos, bem como saber intervir diante dos tais, usando dos produtos e cuidados ideais para reestabelecer a saúde do paciente. Para comunidade científica vai trazer benefício mediante vários aspectos, uma vez que o estudo poderá apresentar dados epidemiológicos, clínicos e terapêuticos que podem abrir outros leques de estudos nesta área carente de pesquisas.

Considerando-se a necessidade de pesquisas neste tema, este trabalho tem como objetivos: Geral: Descrever o perfil sociodemográfico, clínico e terapêutico de pacientes em pós-operatório de estomas de alimentação de um hospital escola. Específicos: Caracterizar os pacientes conforme dados sociodemográficos; identificar as principais indicações, técnica operatória utilizada, tipos de sondas empregadas e dietas em pacientes em pósoperatório de estomas de alimentação; reconhecer as complicações pós-operatórias e o desfecho de pacientes submetidos a estomas de alimentação.

\section{MÉTODO}

A presente pesquisa faz parte de um estudo maior intitulado "Complicações de feridas operatórias em pacientes cirúrgicos" de caráter documental-descritivo, com abordagem quantitativa. A pesquisa foi realizada num Hospital Escola de Campina Grande. O estudo foi desenvolvido por meio da pesquisa em prontuários das clínicas do referido hospital, com busca dos dados a partir de um instrumento de coleta de dados construído pela pesquisadora, contendo características sociodemográficas e clínicas, neste, foram incluídas perguntas com perfil sociodemográfico, clínico e terapêutico, já que muitas condições clínicas envolvem um conjunto de fatores predisponentes, que foram preenchidas conforme análise dos prontuários. Foram selecionados aqueles dentro do intervalo de janeiro de 2012 a dezembro de 2017. O período de realização da coleta foi de dois meses (janeiro à fevereiro de 2018), cujas informações foram transcritas no instrumento de coleta. Os critérios de inclusão foram os prontuários que apresentassem: Pacientes acima de 18 anos; tipo de cirurgia (Gastrostomia ou Jejunostomia); e como critério de exclusão: Prontuários com conteúdo ilegível ou em branco. Para o cálculo do tamanho da amostra de pessoas internadas nas Clínicas do Hospital, realizouse um levantamento do número pessoas com gastrostomias ou jejunostomia internadas durante o período acima, que foi de 93 pessoas. Assim, considerando o cálculo amostral para população finita, com nível de confiança de 95\%, obteve-se uma amostra de 75 prontuários, dos quais, 42 constituíram os dados desta pesquisa, que foram apresentados e analisados. Conforme aprovação pelo Comitê de Ética em Pesquisa, os prontuários foram solicitados ao setor responsável. Os dados foram transferidos para o software https://doi.org/10.31011/reaid-2021-v.95-n.34-art.999 Rev Enferm Atual In Derme v. 95, n. 34, 2021 e-021047 
Excel para construção de uma planilha de dados para as questões contidas nos instrumentos de coleta dos dados. As informações contidas no banco de dados foram transferidas para o pacote estatístico SPSS (Statistical Package for Social Sciences) - versão 21.0. Para análise dos dados, foram obtidas distribuições absolutas e percentis e as medidas estatísticas média e desvio padrão de técnicas de estatística descritiva. A pesquisa foi realizada de acordo os preceitos éticos propostos pela Resolução do Conselho Nacional de Saúde, 466 de 2012, respeitando-se todos os aspectos éticos da pesquisa que envolve seres humanos, seja a instituição ou profissionais. Desse modo, esta pesquisa foi aprovada pelo Comitê de Ética sob CAEE $\mathrm{n}^{\mathrm{o}} \quad$ 57917316.4.0000.5182 e número do Parecer 1.718.517.

\section{RESULTADOS E DISCUSSÃO}

Para melhor compreensão dos resultados desta pesquisa, os dados foram agrupados em oito categorias: 1.Dados sociodemográficos dos pacientes em pósoperatório de estomas de alimentação; 2.Indicação da gastrostomia e jejunostomia; 3.Condições clínicas para indicação da gastrostomia e Jejunostomia; 4.Cuidados no pré-operatório; 5.Técnica operatória utilizada; 6.Tipo de sonda empregada e complicações pós-operatórias dos pacientes com gastrostomia e jejunostomia; 7.Tipo de dieta no pós-operatório; 8.Tempo de hospitalização e desfecho do paciente.

\section{Dados sociodemográficos dos pacientes em pós-operatório de estomas de alimentação}

De acordo com os dados sociodemográficos, o público mais acometido foi o masculino (69\%), pardos $(73,8 \%)$, casados (40,5\%), com baixo nível de escolaridade $(28,6 \%)$, sendo aposentados $(38,1 \%)$ ou agricultores $(31,0 \%)$, com residência na zona urbana $(88,1 \%)$, todos em casa de alvenaria, a maioria não constava religião $(78,6 \%)$, dos que constavam $16,7 \%$ eram católicos, na maior parte dos prontuários a renda também não foi especificada $(64,3 \%)$. Nos que havia parecer da assistência social, 9,5\% tinham renda familiar menor que um salário mínimo; $14,3 \%$ de 1-2 salários mínimos, 9,5\% de 3-4 salários, e 2,4\% mais de 4 salários, ou seja, dos que constavam, a maioria era de 1-2 salários mínimos. Conforme a idade, esta variou de 18-93 anos, com média de 59,9 anos e desvio padrão de 19,6, o que aponta a realização de gastrostomia/jejunostomia em pessoas com idade mais avançada.

A maior prevalência de casos acontece com público masculino. Um estudo realizado com pacientes submetidos a estomas de alimentação revelou que cerca de $88 \%$ eram homens, com a média de idade de 58,4 anos, idade mínima e máxima de 19-87 anos 
respectivamente ${ }^{(2)}$, dados que se aproximam com os desta pesquisa.

As Diretrizes para a vigilância do câncer relacionado ao trabalho do INCA (2013), aponta que a ocupação influencia no desenvolvimento de comorbidades, e o trabalho na agricultura é um dos fatores de risco para o desenvolvimento de câncer de esôfago e estômago devido à exposição a agentes tóxicos e a vapores de combustíveis fósseis. Fatores genéticos, alimentação inadequada, rica em sal, por exemplo, contribuem para o desenvolvimento do câncer gástrico e esofágico, enquanto uma alimentação rica em verduras, legumes e frutas reduzem tal risco, pois os mesmos contêm substâncias oxidantes como vitamina C, fibras, entre outras que possuem essa função preventiva ${ }^{(3-4)}$.

Determinantes sociais, como renda, ocupação/ou profissão, condições de moradia, alimentação, nível de instrução, tabagismo e etilismo, entre outros, contribuem para o aparecimento de diversas comorbidades. Estudo avaliando o perfil dos pacientes com câncer gástrico, também evidenciou ser comum no público masculino, e que o nível de escolaridade em cerca de $69 \%$ era baixo ${ }^{(5-}$ 6). Neste estudo, pelo menos $38,1 \%$ dos pacientes eram ex-fumantes e $31 \%$ eram exetilistas, o que aumenta a predisposição ao adoecimento, haja vista que são hábitos inadequados de vida que poderiam ser mudados e com isso colaborar com redução de diversas comorbidades.

De acordo com o Ministério da Saúde (2014), considera-se uso abusivo de álcool ingestão de cinco ou mais doses de bebida alcoólica para homens e quatro ou mais doses para mulheres, em uma única ocasião, nos últimos 30 dias. Dados da Pesquisa Nacional de Saúde (2013) apontam que o consumo de álcool é mais comum em adultos jovens (1829 e 30-39 anos), com uma prevalência de $21,6 \%$ para homens, enquanto que para mulheres é de $6,6 \%$, constatando-se frequente uso em indivíduos pretos e indígenas, nos fumantes, nos que consideram a saúde boa ou muita boa e também nos que residem na zona urbana $^{(7)}$. O que pode justificar a prevalência deste tipo de câncer na população masculina de acordo com diversas outras pesquisas.

Diante disso, as políticas públicas que visam a redução do consumo do álcool têm grande relevância, uma vez que contribuiria na redução das doenças crônicas não transmissíveis, meta lançada globalmente pela Assembleia Mundial de Saúde de 2015-2025, incluindo entre outros fatores de risco, a redução do tabagismo e etilismo. No Brasil, porém encontra-se uma grande dissociabilidade de interesses, já que, é um ramo comercial que contribui para economia do país, e por isso é amplamente aceito e $\operatorname{comum}^{(7)}$. 


\section{Indicação da Gastrostomia ou Jejunostomia}

Dos 42 pacientes que foram submetidos ao estoma, 37 foi para alimentação, enquanto os demais foi para descompressão gástrica. A gastrostomia ou jejunostomia para descompressão gástrica ou intestinal é utilizada como meio alternativo para drenagem de algum conteúdo em casos de obstrução gástrica ou intestinal por doença maligna inoperável, a exemplo de câncer pancreático, carcinomatose peritoneal, câncer colorretal ou gástrico $^{(8)}$

\section{Condições clínicas para indicação do procedimento cirúrgico}

A tabela 01 são apresentadas as condições clínicas que levaram a realização da gastrostomia ou jejunostomia.

Tabela 01- Condições clínicas que indicaram a cirurgia de Gastrostomia/Jejunostomia, Campina Grande, PB, Brasil, 2018.

\begin{tabular}{lcc}
\hline Variável & $\mathrm{n}$ & $\%$ \\
\hline Dificuldade de deglutição por & 12 & 28,6 \\
$\begin{array}{l}\text { condições neurológicas } \\
\text { Obstrução luminal por doença }\end{array}$ & 12 & 28,6 \\
maligna & 6 & 14,3 \\
Neoplasia de esôfago & 6 & 14,3 \\
Mais de uma condição & 3 & 7,1 \\
Impossibilidade de receber aporte & & \\
calórico por VO à longo prazo & 2 & 4,8 \\
Neoplasia de cabeça e pescoço & 1 & 2,4 \\
Estenose esofágica & 42 & 100,0 \\
\hline TOTAL & & \\
\hline
\end{tabular}

Fonte: Dados da pesquisa, 2018.

Da obstrução luminal por doença maligna, $28,6 \%$ destes eram diagnosticados por diversificados tipos de câncer gástrico. dos 42 participantes, e estavam diagnosticados como: Neoplasia maligna de antro pilórico (1), adenocarcinoma gástrico tubular moderamente diferenciado adenocarcinoma gástrico de células em anel de sinete (1), adenocarcinoma antral (1), adenocarcinoma gástrico com metástase (2), Neoplasia-gástrica-cárdia Borrman IV (1), Neoplasia gástrica Borrman II (1), Neoplasia 
gástrica Borrman III (1), Neoplasia gástrica Borrman IV-T4N2M1 (1), adenocarcinoma gástrico em antro Barrman III (1), adenocarcinoma tubular de corpo gástrico (1).

$\mathrm{Da}$ dificuldade de deglutição por condições neurológicas em 28,6\% estavam presentes as seguintes condições: Esclerose Lateral Amiotrófica-ELA (02), Traumatismo Crânio encefálico- TCE (04), Acidente Vascular Encefálico Hemorrágico/ isquêmico (06).

Dos $06(14,3 \%)$ participantes que tinham câncer de esôfago, foram identificados pelo menos seis tipos diferentes de câncer. Destes, apenas em dois foi realizada jejunostomia, e nos demais, realizadas gastrostomia endoscópica percutânea (02) gastrostomia cirúrgica (02).

Outra condição foi a impossibilidade de receber aporte calórico por VO à longo prazo $(7,1 \%)$, cujas comorbidades presentes eram: Parkinson (01); paralisia cerebral (01); quadro clínico prolongado na UTI associado a outras condições (01). Nos outros $14,3 \%$ estavam presentes mais de uma condição que indicou a necessidade do suporte enteral, e outras $4,8 \%$ estavam relacionadas à neoplasia de cabeça $\mathrm{e}$ pescoço.

De Acordo com estimativa do INCA, em 2020, no Brasil, o câncer de estômago ocupa o $4^{\circ}$ lugar entre os 10 tipos de cânceres mais incidentes, estima-se que novos casos de câncer de estômago para 2020, 2021 e 2022 é de 13.360 em homens e 7.870 nas mulheres, mostrando assim, ser uma causa comum em ambos os sexos, que podem favorecer a realização destes dois tipos de cirurgia para suporte nutricional.

Um estudo mostra resultados semelhantes ao desta pesquisa, onde, das indicações clínicas para realização da gastrostomia, $76,1 \%$ eram as condições neurológicas, enquanto que as condições neoplásicas representaram a segunda causa para realização do procedimento $^{(9)}$.

\section{Cuidados no pré-operatório}

Na Tabela 02 são demonstrados os cuidados que foram analisados nos pacientes submetidos à estomas de alimentação.

Tabela 02: Cuidados da pele no pré-operatório e intra-operatório em pacientes submetidos à estomas de alimentação, Campina Grande, PB, Brasil, 2018.

\begin{tabular}{lrl}
\hline Variável & $\mathrm{n}$ & $\%$ \\
\hline Tricotomia & 26 & 62 \\
Não & 16 & 38 \\
Não Consta &
\end{tabular}




\begin{tabular}{lll} 
Não Consta & 24 & 57,1 \\
Sim & 18 & 42,9 \\
Banho & & \\
Não Consta & 25 & 59,6 \\
Sim & 17 & 40,4 \\
\hline TOTAL & 42 & 100,0
\end{tabular}

Fonte: Dados da pesquisa, 2018.

É importante observar que informações como banho, antissepsia da pele no centro cirúrgico não estavam documentados, e, portanto, é uma problemática que necessita ser reavaliada em todo contexto assistencial, tanto pelo profissional de enfermagem quanto pelo profissional médico, pois o registro correto das informações é necessário no âmbito do cuidado. Estes são cuidados básicos que colaboram para redução de complicações cirúrgicas, tendo em vista que o número de procedimentos cirúrgicos tem aumentando concomitante à atualização das técnicas operatórias, estimando-se que sejam realizadas de 187-281 milhões de cirurgias, o que representa uma cirurgia a cada 25 indivíduos. Em contrapartida, observam-se diversas complicações, variando de $3-16 \%$, e óbitos de 5-10\% em países subdesenvolvidos, sendo um número agravante que requer atenção tanto por estes danos como pelo custo aos cofres públicos ${ }^{(10)}$.

Um estudo que monitorizou a colonização bacteriana orofaríngea de 274 pacientes submetidos a PEG naqueles com distúrbios neurológicos e com neoplasia, identificou 14 tipos de microrganismos através de swabs orofaríngeos. Em 57\% dos pacientes o mesmo microorganismo foi encontrado tanto na orofaringe quanto periestomal, o qual causara infecção local, concluindo que a triagem microbiana pode ser um marcador de infecção periestoma, uma complicação comum em pacientes oncológicos $^{(11)}$. Portanto, cuidados perioperatórios são de extrema importância para redução desses índices, como o banho, a higiene oral com clorexidina à $0,12 \%$, entre outros cuidados que são imprescindíveis para redução de complicações ${ }^{(10)}$. Embora, estudo não evidencie a eficácia do uso das soluções de gluconato de clorexidina 4\%, polivinilpirolidona iodo (PVP-I) $10 \%$ degermante ou sabão sem antisséptico durante o banho para prevenção de infecção do sítio cirúrgico, este é um cuidado recomendável no pré-operatório $^{(12)}$.

\section{Técnica operatória utilizada}

As técnicas operatórias utilizadas foram: Gastrostomia Endoscópica PercutâneaPEG 19 (45,2\%), Jejunostomia 15 (35,7\%) e Gastrostomia cirúrgica 08 (19,0\%). A PEG é a mais difundida, por apresentar menor custo https://doi.org/10.31011/reaid-2021-v.95-n.34-art.999 Rev Enferm Atual In Derme v. 95, n. 34, 2021 e-021047 
e menores complicações aos pacientes, por ser menos invasiva, causar menor trauma cirúrgico, em relação à outras técnicas de inserção de sondas para alimentação ${ }^{(13)}$.

\section{Tipo de sonda empregada e complicações pós-operatórias dos pacientes com gastrostomia e jejunostomia}

Os tipos de sondas utilizadas foram: sonda do tipo Foley 18 (43\%), sonda de silicone 10 (23,8\%), sonda Nelaton 04 (9,5\%), sonda Mickey 01 (2,3\%), enquanto em 9 prontuários $(21,4 \%)$ não tinha descrição do tipo de sonda empregada.

Na Tabela 03 são expostos os números e a frequência das complicações presentes nos pacientes submetidos a gastrostomia e jejunostomia.

Tabela 03: Complicações no pós-operatório de estomas de alimentação, Campina Grande, PB, Brasil, 2018.

\begin{tabular}{lll}
\hline \multicolumn{1}{c}{ Variável } & $\mathrm{n}$ & $\%$ \\
\hline Dor & 22 & 52,4 \\
\hline Outras complicações & 18 & 42,9 \\
\hline Presença de exsudato & 15 & 35,7 \\
\hline Dermatite & 14 & 33,3 \\
\hline Infecção periestomal & 10 & 23,8 \\
\hline Hemorragia & 9 & 21,4 \\
\hline Vazamento da dieta & 7 & 16,7 \\
\hline Broncoaspiração & 3 & 7,1 \\
\hline Migração da Sonda & 2 & 4,8 \\
\hline Perda precoce da sonda & 2 & 4,8 \\
\hline Peritonite & 2 & 4,8 \\
\hline Queimadura & 2 & 4,8 \\
\hline periestomal & & 4,8 \\
\hline Desabamento da sonda & 2 & 2,4 \\
\hline Edema periestomal & 1 & 100,0 \\
\hline TOTAL & 42 & \\
\hline
\end{tabular}

Fonte: Dados da pesquisa, 2018.

As complicações foram mais frequentes naqueles pacientes que utilizaram sonda do tipo foley (dispositivo de inserção uretral que https://doi.org/10.31011/reaid-2021-v.95-n.34-art.999 Rev Enferm Atual In Derme v. 95, n. 34, 2021 e-021047

é utilizado para gastrostomia/jejunostomia), estando presentes pelo menos $15(35,7 \%)$ complicações: dor, hemorragia, presença de (i) 
fecalóide, presença de exsudato periestomal, deiscência da Ferida Operatória, dermatite, peritonite, queimadura periestomal, infecção periestomal, edema da parede abdominal, sepse, vazamento da dieta ao redor da sonda, êmese frequente, broncoaspiração e insuficiência respiratória aguda. Vale ressaltar que cada paciente possuía ou uma ou mais destas complicações, umas mais graves e outras menos complexas. Do total dos pacientes com sonda de Foley (18 pacientes), apenas três não tiveram complicações.

A sonda de silicone também demonstrou várias complicações, que foram: vazamento da dieta ao redor da sonda, desabamento da sonda, presença de exsudato ao redor da sonda, infecção periestomal, broncoaspiração, refluxo gastroesofágico, dermatite periestomal, dor intensa, deiscência da Ferida operatória, reabordagem cirúrgica da gastrostomia. Sendo assim, do total de dez pacientes com sonda de silicone, pelo menos quatro tiveram estas complicações, e os demais (seis pacientes) não tiveram.

Dos pacientes com sonda de Nelaton também estiveram presentes: diarreia, infecção periestomal, dermatite periestomal, náuseas e vômito fecalóide, dor periestomal, presença de exsudato. Apenas um paciente não teve complicações. Mesmo naqueles em que não havia documentado o tipo de sonda, oito deles tiveram complicações. E em um paciente que foi colocada a sonda Mickey houve complicações e foi inserida a sonda Foley.

Sondas do tipo Foley são causadoras de muitas complicações. Os tubos PEG padrão (que possuem o anel externo/ para-choque) evita que a sonda migre completamente para o estômago e duodeno, diferentemente da sonda de Foley, que não possui, porém ainda é frequentemente usada devido acessibilidade financeira $^{(14)}$, no entanto estudos revelam que pacientes que utilizam esta sonda apresentam múltiplas complicações, e concluem que seu uso deve ser abolido na prática clínica devido as múltiplas intervenções que poderiam ser evitadas nestes pacientes ${ }^{(15-16)}$.

A dor é um sintoma subjetivo e causa estresse psicológico e físico. As gastrostomias abertas ou laparoscópicas têm sido relacionadas à complicação do tipo dor pósoperatória quando comparadas com outras técnicas de passagem de sonda, porém em pacientes com estenose grave, câncer de cabeça e pescoço, doença maligna do esôfago, estas técnicas são indicadas ${ }^{(17)}$.

\section{Tipo de dieta administrada no pós-operatório}

Pelo menos 10 tipos de dieta foram identificados nos prontuários, cada uma delas estavam descritas no primeiro item da prescrição médica, atualizada diariamente conforme rotina hospitalar. O tipo de dieta mais comum foi a líquida de prova $(52,4 \%)$, solução glicosada a 5\% (14,3\%) diretamente 
na gastrostomia ou jejunostomia, uma pequena parcela representa a dieta de solução fisiológica a $0,9 \%(4,8 \%)$ e as demais foram variadas, sendo: dieta sem resíduos, para hipertenso, para hipertenso e diabético, hiperprotéica e/ou hipercalórica, hipoglicídica, hiperprotéica e/ou hipercalórica, dieta livre; apenas um paciente não fez dieta e em três prontuários não constavam nenhuma dieta. O período de início da dieta variou entre 1 hora (tempo de início mínimo) e 192 horas (tempo máximo).

Persistem as diferentes ideologias com relação a introdução da dieta no pósoperatório, e assim muitos protocolos institucionais são desenvolvidos de acordo com suas realidades. De acordo com o Protocolo ACERTO (Aceleração da Recuperação Total Pós-operatória), o retorno do trânsito gastrointestinal difere em cada parte anatômica do Trato gastrointestinal: o do estômago retorna em 24 horas, intestino delgado 5 a 7 horas, o cólon direito de 24 a 48 horas e o colón esquerdo de 36 a 60 horas. Assim, pacientes que são submetidos a cirurgias gastrointestinais, oferta-se dieta líquida no mesmo dia, desde que não apresente vômitos e que haja boa aceitação ${ }^{(18)}$. Um estudo realizado com 117 pacientes submetidos a PEG, avaliando o tipo de introdução alimentar no pós-operatório, em dieta líquida e semi-sólida, concluíram que a administração da dieta semi-sólida em quase metades destes pacientes, contribuiu para redução de eventos adversos, principalmente o refluxo gastroesofágico e consequentemente de pneumonia por broncoaspiração. Notou-se ainda, que o tempo de internação foi reduzido nos que usaram dieta semissólida, além de uma menor taxa de readmissão hospitalar, indicando que pode ser alternativa nutricional de primeira escolha ${ }^{(19)}$.

\section{Tempo de hospitalização e desfecho dos pacientes}

O tempo de internação dos pacientes para cirurgia variou de $1 \mathrm{~h}$ (tempo mínimo), a 720 horas (equivalente à 30 dias), com uma média de 189,76 horas, equivalente a 7,9 dias com desvio padrão de 193,6, indicando um tempo de internação parecido entre a maioria dos pacientes.

O tempo de internação no pósoperatório destes pacientes variou de menos de um dia (tempo mínimo) após a troca da gastrostomia, ou de 03 dias a um mês, estes últimos representando uma taxa de $54,8 \%$, ou seja, a maioria teve internação prolongada. Outros 14,3\% tiveram tempo de internação de 1-2 meses, estando associado a complicações após o procedimento.

O tempo prolongado de internação no pós-operatório pode estar relacionado a vários fatores, tanto por já estarem internados em outras clínicas do referido hospital, e só a partir da estabilização do quadro crítico realizar a cirurgia ou de receber o diagnóstico definitivo para poder realizá-la, o que https://doi.org/10.31011/reaid-2021-v.95-n.34-art.999 Rev Enferm Atual In Derme v. 95, n. 34, 2021 e-021047 
demandou maior tempo de internação no préoperatório, que pode ter repercutido no pós; à administração da dieta tardiamente; como também ao quadro clínico dos pacientes, tendo em vista que a maioria deles eram portadores de câncer $(52,4 \%)$ ou já eram admitidos com quadro de desnutrição $(52,4 \%)$. Pacientes com doenças crônicas subjacentes, pacientes com doença maligna, e pacientes idosos podem ser susceptíveis a complicações pós-operatórias, com maior risco para mortalidade quando submetidos a $\mathrm{PEG}^{(20)}$

O desfecho dos pacientes foi alta hospitalar após a inserção da gastrostomia/jejunostomia $(66,7 \%)$; readmissão e alta foi $11,9 \%$; readmissão e óbito foram 4,8\%, a maioria devido complicações do estado clínico geral; e 16,7\% foram a óbito. Destes óbitos, cinco foram causados por sepse, uma complicação grave após gastrostomia/jejunostomia.

Pode-se considerar que o número de mortes por sepse no público avaliado neste estudo foi elevado devido à população ser pequena, pois de um total de 42 pacientes, $11,9 \%$ morreram de sepse. Verifica-se assim que as causas para mortalidade de pacientes submetidos à gastrostomia/jejunostomia estão relacionadas às complicações tanto locais quanto sistêmicas, e que condições graves como a sepse podem ocorrer com grande frequência, necessitando de medidas assistenciais efetivas que colaborem para preveni-la.

\section{CONCLUSÃO}

A avaliação do perfil epidemiológico, clínico e terapêutico dos pacientes submetidos à gastrostomia ou jejunostomia ajudou a esclarecer melhor o seu manejo terapêutico. A maioria dos pacientes foi do sexo masculino, com hábito de ex-etilismo ou ex-tabagismo, que também são fatores que predispõem as comorbidades que indicaram a realização do procedimento, as quais foram predominantemente o câncer gástrico ou câncer de esôfago, já em estágio avançado.

$\mathrm{O}$ procedimento de gastrostomia endoscópica foi o procedimento comumente realizado nos pacientes no período de tempo analisado, e não diferente de outros estudos. A eficácia desta conduta tem melhorado o desfecho clínico dos pacientes, devido ser um procedimento menos invasivo do que a gastrostomia cirúrgica. Porém, mesmo diante desta prática que ajuda a reduzir os danos aos pacientes, ainda foi possível identificar alta taxa de morbidade, conforme se evidenciou pela presença de muitas complicações pósoperatórias relacionadas à inserção do tubo para alimentação, onde cerca de treze complicações de menor gravidade e outras de maior gravidade foram notadas.

O tipo de sonda utilizada também pode ser um fator predisponente para desenvolvimento das complicações, tendo em 
vista que a mais utilizada foi a Sonda de Foley, dispositivo de inserção uretral, mas que é amplamente utilizado como tubo para alimentação em pacientes gastrostomizados ou jejunostomizados. Como o maior índice de complicações foram relacionadas ao tubo, a alterações locais da pele periestoma, e também sistêmica, sugere-se que seu uso seja uma prática menos corriqueira, pois a acessibilidade financeira que o produto oferece em comparação as outras sondas indicadas para alimentação na gastrostomia ou jejunostomia é temporária, tendo em vista que num período curto de pós-operatório já são verificadas diversas complicações, e tal uso pode limitar a utilização da sonda de Foley. Vale ressaltar que os outros tipos de sonda também trouxeram estes riscos, a exemplo da sonda de silicone, mas que o índice de complicação dos pacientes que a utilizaram foi menor e o benefício foi bem expressivo, pois pelo menos seis deles não tiveram nenhuma complicação.

\section{REFERÊNCIAS}

1. Lucendo AJ, Friginal-Ruiz AB. Percutaneous endoscopic gastrostomy: An update on its indications, management, complications, and care. Rev esp enferm dig. [Internet]. 2014 [acesso em 24 de out 2020]; 106 (8):529-539. Disponível em: https://pubmed.ncbi.nlm.nih.gov/2554 $\underline{4410 /}$
A identificação de diversos fatores pôde permitir a conclusão de que as complicações pós-operatórias são de origem multivariada, pois envolve a doença de base do paciente, o nível de desnutrição, os hábitos de vida atuais ou pregressos, a hereditariedade, o tempo internação no pré e pós-operatório, o tempo de início da dieta/o tempo de jejum que o paciente enfrenta que gera estresse fisiológico; o trauma relacionado ao procedimento cirúrgico e a capacidade do indivíduo de reagir a este estresse, ao tipo de sonda empregada, aos cuidados de enfermagem tanto pré quanto no pósoperatório. Todos são indicativos de qual desfecho o paciente vai receber, seja alta, readmissões hospitalares para tratar as complicações, ou óbito quando estas não são revertidas. Identificou-se alta frequência da ausência de informações importantes nos prontuários dos pacientes, o que pode prejudicar a assistência e dificultar pesquisas, uma vez que interfere na avaliação de diversos indicadores.

2. Anselmo CA, Tercioti VJ, Lopes LR, Souza JCN, Andreollo NA. Gastrostomia cirúrgica: indicações atuais e complicações em Gastrostomia cirúrgica: indicações atuais e complicações em pacientes de um hospital universitário. Rev Col Bras Cir [Internet]. 2013 [acesso em 24 de out 2020]; 40: 458-46. Disponível em: https://www.scielo.br/scielo.php?scrip $\mathrm{t}=$ sci_arttext\&pid=S010069912013000600007 
3. Mota OM, Curado MP, Oliveira JC, Martins E, Cardoso DMM. Risk factors for esophageal cancer in a lowincidencearea of Brazil. Med J. [Internet]. 2013[acesso em 24 de out 2020]; 131(1):27-34. Disponível em: https://www.scielo.br/scielo.php?scrip $\mathrm{t}=\mathrm{sci}$ arttext\&pid=S1516$\underline{3180201300010002}$

4. Ferrari F. Estudo dos Fatores de Risco associados ao câncer gástrico pela análise de bancos de dados populacionais [Monografia]. Joinville: Universidade da Região de Joinville; 2013.

5. Ministério da Saúde (BR). Determinantes sociais, econômicos e ambientais da saúde. A saúde no Brasil em 2030 - prospecção estratégica do sistema de saúde brasileiro: população e perfil sanitário. Rio de Janeiro. 2013; 2: 19-38.

6. Carvalho FLN. Análise do perfil clínico, epidemilógico e histopatológico do câncer gástrico na população de Roraima [Dissertação]. Roraima: Universidade Federal de Roraima; 2016.

7. Garcia LP, Freitas LRS. Consumo abusivo de álcool no Brasil: resultados da Pesquisa Nacional de Saúde 2013. Epidemiol. Serv. Saúde [Internet]. 2015 [acesso em 24 de out 2020]; 24(2): 227-237. Disponível em: http://scielo.iec.gov.br/scielo.php?scri $\mathrm{pt}=$ sci_arttext\&pid=S1679$\underline{49742015000200005}$

8. Kawata N, Kakushima N, Tanaka M, Sawai H, Imai K, Hagiwara T, Takao T, Hotta K, Yamaguchi Y, Takizawa $\mathrm{K}$, Matsubayashi $\mathrm{H}$, Ono $\mathrm{H}$.
Percutaneous endoscopic gastrostomy for decompression of malignant bowel obstruction. Dig Endosc [Internet].2014 [acesso em 24 de out 2020]; 26(2):208-13. Disponível em: https://www.researchgate.net/publicati on/239945587_Percutaneous_endosco pic_gastrostomy_for_decompression of_malignant_bowel_obstruction

9. Ferreira Filho J. Ampliação do acesso à gastrostomia endoscópica por meio da revisão de práticas gerenciais e operacionais [Dissertação]. Ribeirão Preto: Universidade de São Paulo; 2015.

10. Ministério da Saúde (BR). Anvisa. Medidas de Prevenção de Infecção Relacionada à Assistência à Saúde. Brasília; 2017.

11. Kroupa R, Jurankova J, Dastych M, SenkyrikM, Pavlik T, Prokesova J,Jecmenova, Dolina J, Hep A. Different Clinical Utility of Oropharyngeal Bacterial Screening prior to Percutaneous Endoscopic Gastrostomy in Oncological and Neurological Patients. Biomed Res Int [Internet]. 2014 [acesso em 24 de out 2020]; 2014: 59089. Disponível em: https://www.ncbi.nlm.nih.gov/pmc/art $\underline{\text { icles/PMC4163457/ }}$

12. Franco LMC, Almeida AGI, Duarte GMH, Lamounier L, Pinto TS, Pereira PFS, Chianca TCM, Ercole FF. Efeitos do banho pré-operatório na prevenção de infecção cirúrgica: estudo clínico piloto. Rev Min Enferm [Internet]. 2017 [acesso em 24 de out 2020]; 21:e-1053. Disponível em: https://pesquisa.bvsalud.org/portal/res ource/pt/bde-32207 
13. Rahnemai-Azar AA, Rahnemaiazar AA, Naghshizadian R, Kurtz A, Farkas DT. Percutaneous endoscopic gastrostomy: Indications, technique, https://pubmed.ncbi.nlm.nih.gov/2497 $\underline{6711 /}$

14. Fonseca J, Nunes G, Patita M, Barosa $\mathrm{R}$, Santos CA. Catheter traction and gastric outlet obstruction: a repeated complication of using a Foley catheter for gastrostomy tube replacement. Nutr Hosp [Internet].2017 [acesso em 24 out 2020]; Mar 30;34(2):499-501. Disponível em: https://pubmed.ncbi.nlm.nih.gov/2842 $\underline{1810 /}$

15. Metussin A, Sia R, Bakar S, Chong VH. Foley Catheters as Temporary Gastrostomy Tubes: Experience of a Nurse-Led Service. Gastroenterol Nurs[Internet]. 2016 [acesso em 24 out 2020]; 39(4):273-7. Disponível em:

tps://pubmed.ncbi.nlm.nih.gov/274670 $58 /$

16. Ojo, O. Problems with use of a Foley catheter in enteral tube feeding. $\mathrm{Br} \mathbf{J}$ Nurs [Internet] 2014 [acesso em 24 out 2020]; 23(7):360-2, 364. Disponível em: https://pubmed.ncbi.nlm.nih.gov/2473 2987/ complications and management. World $\mathbf{J}$ of Gastroenterol [Internet]. 2014 [acesso em 24 out 2020] 20(24): 7739-7751. Disponível em:

17. Mizrahi I, Garg H, Divino CM, Nguyen S. Comparison of Laparoscopic Versus Open Approach to Gastrostomy Tubes. JSLS[Internet].2014 [acesso em 24 out 2020]; 18 (1): 28-33. Disponível em: https://www.ncbi.nlm.nih.gov/pmc/art icles/PMC3939338/

18. Nascimento JEA. Acerto: Acelerando a recuperação total pós-operatória. 3 ed. Rio de Janeiro. Rubio; 2016.

19. Toh Yoon EW, Yoneda K, Nishihara K. Semi-solid feeds may reduce the risk of aspiration pneumonia and shorten postoperative length of stay after percutaneous endoscopic gastrostomy (PEG). Endoscopy International Open.[Internet].2016 [acesso em 24 out 2020]; 04: E1247E1251.

20. Pih GY, Na HK, Ahn JY, Jung KW, Kim DH, Lee JH, Choi KD, Song HJ, Lee GH, Jung HY. Risk factors for complications and mortality of percutaneous endoscopic gastrostomy insertion. BMC Gastroenterology [Internet]. 2018 [acesso em 24 out 2020]; 18:101. Disponível em: https://bmcgastroenterol.biomedcentra 1.com/articles/10.1186/s12876-0180825-8

Submissão: 2021-01-12

Aprovado: 2021-03-23 